\title{
Wave-like Formation of Hot Loop Arcades
}

\author{
A. Reva ${ }^{1} \cdot$ S. Shestov ${ }^{1} \cdot$ I. Zimovets ${ }^{2}$. \\ S. Bogachev ${ }^{1} \cdot$ S. Kuzin ${ }^{1}$
}

(c) Springer $\bullet \bullet \bullet \bullet$

\begin{abstract}
We present observations of hot arcades made with the Mg XII spectroheliograph onboard the CORONAS-F mission, which provides monochromatic images of hot plasma in the MgXII $8.42 \AA$ resonance line. The arcades were observed to form above the polarity inversion line between Active Regions NOAA 09847 and 09848 at four successive episodes: at 09:18, 14:13, and 22:28 UT on 28 February 2002, and at 00:40 UT on 1 March 2002. The arcades all evolved in the same way: a) a small flare (precursor) appeared near the edge of the still invisible arcade, b) the arcade brightened in a wave-like manner — closer loops brightened earlier, and c) the arcade intensity gradually decreased in $\approx 1 \mathrm{~h}$. The estimated wave speed was $\approx 700 \mathrm{~km} \mathrm{~s}^{-1}$, and the distance between the hot loops was $\approx 50 \mathrm{Mm}$. The arcades formed without visible changes in their magnetic structure. The arcades were probably heated up by the instabilities of the current sheet above the arcade, which were caused by an MHD wave excited by the precursor.
\end{abstract}

Keywords: Flares, Waves

\section{Introduction}

It is generally accepted that the energy source of solar flares is the energy of the coronal magnetic field. According to the standard flare model (Carmichael, 1964; Sturrock, 1966; Hirayama, 1974; Kopp and Pneuman, 1976), a magnetic reconnection occurs in the corona. Inside the reconnection region, electrons are accelerated. The accelerated electrons propagate to the lower layers of the solar atmosphere, stop in the chromosphere, heat it, and create hard X-ray (HXR) sources. The heated chromoshperic material expands into the corona filling the magnetic field lines with hot and dense plasma. Many flares show signs of the standard model: HXR emission at the loop footpoints and apexes, plasma upflows inferred from Doppler shifts, and magnetic field configuration highlighted in extreme ultra-violet (EUV) images.

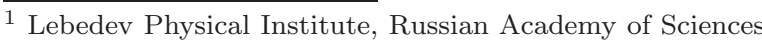
email: reva.antoine@gmail.com

2 Space Research Institute, Profsoyuznaya 84/32, Moscow 117997, Russia
} 
Post-flare arcades are a good testing ground for the standard flare model. Whereas arcades are frequently observed with EUV telescopes, a 'truly hot' arcade is not as frequent. In fact, the only report we found is given by Warren et al. (1999). Slightly more numerous are observations of HXR ribbons in the arcade footpoints (Masuda, Kosugi, and Hudson. 2001: Liu et al., 2007; Jing et al., 2007; Krucker et al., 2011). In all these articles, the authors stressed that the properties of the phenomena under analysis $-\mathrm{H}_{\alpha}$ and $\mathrm{HXR}$ ribbons, magnetic reconfiguration, sheared magnetic field, and heating and cooling of plasmas - are consistent with the standard flare model.

The standard flare model is $2 \mathrm{D}$ or $2.5 \mathrm{D}$ in nature. However, some observations require a $3 \mathrm{D}$ explanation; for example, sequential brightening of the flare arcade (Vorpahl, 1976), spreading of heating from a localized flare to a neighboring active region (Parenti. Reale, and Reeves, 2010), HXR footpoint motion along the arcade (Bogachev et al., 2005; Grigis and Benz, 2005), and asymmetric filament eruption (Liu. Alexander, and Gilbert, 2009; Tripathi, Isobe, and Mason, 2006). To further develop a flare theory, we need to extend the standard flare model into 3D.

It is widely accepted that changes in the coronal magnetic structure - flux emergence, flux cancellation, or footpoints shearing motion - may drive flare reconnection (see reviews such as Benz, 2008; Fletcher et al., 2011). However, several authors have proposed that MHD waves can trigger instabilities in the current sheet, which will lead to the reconnection (Vorpahl, 1976: Somov and Syrovatskii, 1982; Nakariakov et al., 2006; McLaughlin. Hood, and de Moortel, 2011; Nakariakov and Zimovets, 2011; Artemyev and Zimovets, 2012).

To observationally investigate flare heating, we need some way to see hot plasma. The most commonly used instruments for hot plasma research - the Atmospheric Imaging Assembly (AIA; Lemen et al., 2011) onboard the Solar Dynamic Observatory (SDO), the X-Ray Telescope (XRT; Golub et al., 2007) onboard Hinode, and the Soft X-ray Telescope (SXT; Tsuneta et al., 1991) onboard Yohkoh - have wide spectral sensitivity. Their images contain cold plasma background, and isolation of hot plasma requires various reasonable assumptions (Reale et al., 2011; Warren. Winebarger, and Brooks, 2012; Testa and Reale, 2012). It would be ideal for flare heating research to obtain monochromatic images of the solar corona in a hot monochromatic line.

In this work, we present the first observations of the formation of hot loop arcades in a hot monochromatic line. The behavior of the observed arcades cannot be explained with the $2.5 \mathrm{D}$ standard flare model: they formed in a wavelike manner without changes in their magnetic structure. The aim of this work is to present the observations and give them a reasonable interpretation.

\section{Observational Data}

We analyzed hot loop arcades observed on 28 February 2002 at 09:18, 14:13, and 22:28 UT and on 1 March 2002 at 00:40 UT. We mainly used the Mg XII spectroheliograph (Zhitnik et al., 2003) data to investigate the behavior of hot arcades, the Michelson Doppler Imager (MDI; Scherrer et al., 1995) data to infer 


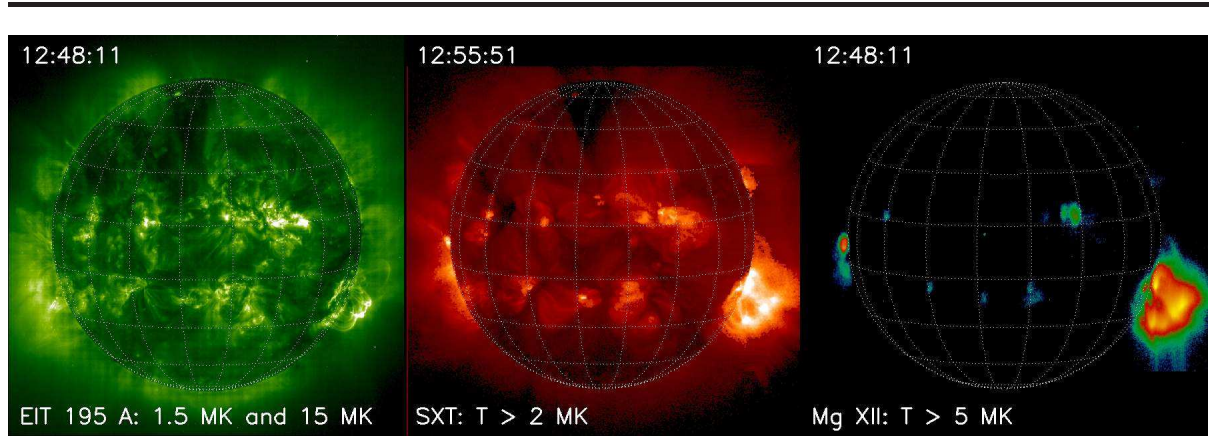

Figure 1. Comparison of hot plasma imagers. Left: EIT $195 \AA$ image, middle: SXT image (AlMg filter), right: Mg XII image. Images were taken on 1 October 2001.

the photospheric magnetic field beneath the arcades, the Reuven Ramaty High Energy Solar Spectroscopic Imager (RHESSI; Lin et al., 2002) data to determine where the accelerated particles were present in the arcade, and the Extreme ultraviolet Imaging Telescope (EIT; Delaboudinière et al., 1995) data to see how the arcades looked like in low-temperature lines.

The Mg XII spectroheliograph was dedicated for hot plasma observations. It was launched in 2001 onboard the CORONAS-F satellite (Oraevsky and Sobelman. 2002) as a part of the SPIRIT instrumentation complex (Zhitnik et al., 2002). The instrument provides monochromatic solar coronal images in the Mg XII $8.42 \AA$ resonance line. The plasma emits this line at temperatures greater than $5 \mathrm{MK}$; therefore, the Mg XII images contain only signal from hot plasma without any low-temperature background. Mg XII images differ from other telescopic images - there is neither a solar limb nor a quiet Sun background (see Figure (1). Typical structures in Mg XII images range from 4 to $300 \mathrm{Mm}$ in size and have a lifetime of several seconds up to several days (Urnov et al., 2007). The Mg XII spectroheliograph has two main advantages over 'traditional' hot coronal imagers: 1) the entire signal shown in the Mg XII images is from a hot plasma, and 2) faint features can be seen, because no cold background obscure them.

In the present work, we used the Mg XII spectroheliograph data obtained with a 105 s cadence and an $8^{\prime \prime}$ resolution. Due to instrumental effects, Mg XII images are slightly elongated in one of the directions (Reva et al., 2012). We pre-processed the Mg XII data by subtracting the background and removing the signal caused by the radiation damage. To improve the visibility of faint features on the Mg XII images, we used a power scale with index 0.3 (see Figures 1- 3). In the Mg XII images of Figures 1-3, blue and green correspond to low intensities, red and yellow to high intensities.

The MDI onboard the SOHO satellite (Domingo, Fleck, and Poland, 1995), maps the line-of-sight component of the photospheric magnetic field in a synoptic mode with a $4^{\prime \prime}$ resolution and a 90 min cadence.

The RHESSI observes HXR spectra from $3 \mathrm{keV}$ up to $17 \mathrm{MeV}$. Using Fourierbased methods, RHESSI can synthesize HXR images in the same spectral range. RHESSI data were available for two out of four observed arcades. 
The EIT on SOHO satellite takes solar images at the wavelengths centered at $171,195,285$, and $304 \AA$. In a synoptic mode, EIT takes images in all four channels every $6 \mathrm{~h}$; in the 'CME watch' mode the telescope takes images in the $195 \AA$ channel every $12 \mathrm{~min}$. The pixel size of the telescope is $2.6^{\prime \prime}$ and the spatial resolution is $5^{\prime \prime}$. We used 'CME watch' EIT data for auxiliary purposes - to observe the cool plasma. Unfortunately, no $H_{\alpha}$ images were available for the analyzed arcades.

\section{Results}

All four hot arcades formed in the same active region (AR), above the polarity inversion line (PIL) between the two elongated regions of opposite polarities (AR NOAA 09847 and 09848; see Figure 2 left). On 25 February 2002, there was a flux emergence in the western part of the negative polarity region, and on 1 March 2002, it disappeared. This flux emergence caused 26 flares, and in four of them we observed the formation of hot loop arcades at 09:18, 14:13, and 22:28 UT on 28 February 2002, and at 00:40 UT on 1 March 2002 (see Figure 3). All four arcades evolved in the same way:

i) A small hot source, which we call 'the precursor', appeared above the flux emergence region in the Mg XII images. At the same place and approximately at the same time, an HXR source appeared in the RHESSI images (see Figure 4). This phase lasted for 2-10 min.

ii) Then, a hot plasma was observed to fill the loops in a wave-like process: the loops that were closer to the precursor structure were filled earlier than the loops that were farther away. All four arcades formed in $5 \mathrm{~min}$, which corresponds to a wave speed of approximately $700 \mathrm{~km} \mathrm{~s}^{-1}$.

iii) The emission intensity of the loops increased and reached maximum after 5 $20 \mathrm{~min}$. The maximum intensity was located in the apex of the loops above the PIL.

iv) The emission intensity of the loops gradually decreased over approximately $1 \mathrm{~h}$.

Table 1 lists the characteristics of the observed arcades.

All four arcades had similar sizes; the loop length was $170 \mathrm{Mm}$, and the arcade length was $200 \mathrm{Mm}$ (see Figure 2 center). The arcades consisted of $3-5$ loops

Table 1. Arcades parameters.

\begin{tabular}{llllllll}
\hline No & Date & $\begin{array}{l}\text { GOES } \\
\text { class }\end{array}$ & $\begin{array}{l}\text { Precursor } \\
\text { start }\end{array}$ & $\begin{array}{l}\text { First } \\
\text { loop } \\
\text { start }\end{array}$ & $\begin{array}{l}\text { Last loop } \\
\text { start }\end{array}$ & $\begin{array}{l}\text { Arcade } \\
\text { faded } \\
\text { away }\end{array}$ & $\begin{array}{l}\text { Number } \\
\text { of loops }\end{array}$ \\
\hline 1 & 28 Feb 2002 & C4.0 & $09: 20$ & $09: 27$ & $09: 29$ & $11: 20$ & 5 \\
2 & 28 Feb 2002 & C5.5 & $14: 13$ & $14: 16$ & $14: 20$ & $15: 45$ & 3 \\
3 & 28 Feb 2002 & C7.5 & $22: 35$ & $22: 40$ & $22: 45$ & $23: 49$ & 3 \\
4 & 01 Mar 2002 & C3.0 & $00: 37$ & $00: 42$ & $00: 49$ & $01: 33$ & 4 \\
\hline
\end{tabular}






Figure 2. The hot loop arcade of 28 February 2002 at 09:23 UT. Left: MDI image (the movie is available as electronic supplementary material); the red contours denote the Mg XII signal. Middle: Mg XII 8.42 A image. Right: EIT $195 \AA$ image; the blue contours denote the Mg XII signal. The coordinates are in units of arcsec.

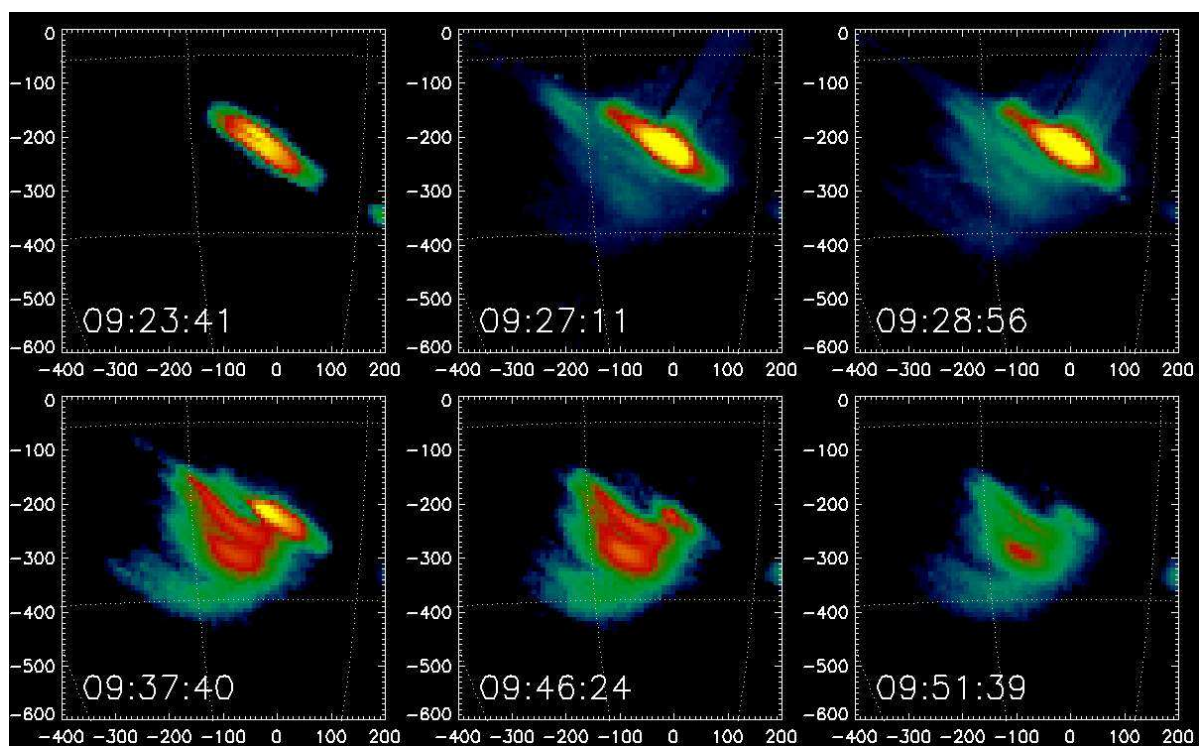

Figure 3. The hot arcade of 28 February 2002 at 09:23 UT; '0' marks the precursor structure and ' 1 ' - ' 5 ' denote the arcade. The coordinates are in units of arcsec. (A movie is available as electronic supplementary material.)

separated at $\approx 50 \mathrm{Mm}$. The brightness of the loops gradually decreased with distance from the precursor structure. The loop footpoints were rooted in the main regions of positive and negative polarities (see Figure 3).

In the EIT $195 \AA$ images, the precursor structure looked like a loop with a size of $20 \mathrm{Mm}$, but the arcade was invisible (see Figure2 2right). When the arcade cooled down and disappeared from the Mg XII images, the brightest loop of the arcade appeared in the EIT $195 \AA$ images.

The HXR source appeared above the flux emergence region at 09:26 and 14:14 UT (events 1 and 2; see Figure 44). There were no other HXR emissions during the events. RHESSI data were unavailable for the other two events. Figure 5 shows RHESSI, GOES, and Mg XII light curves for the arcade that 

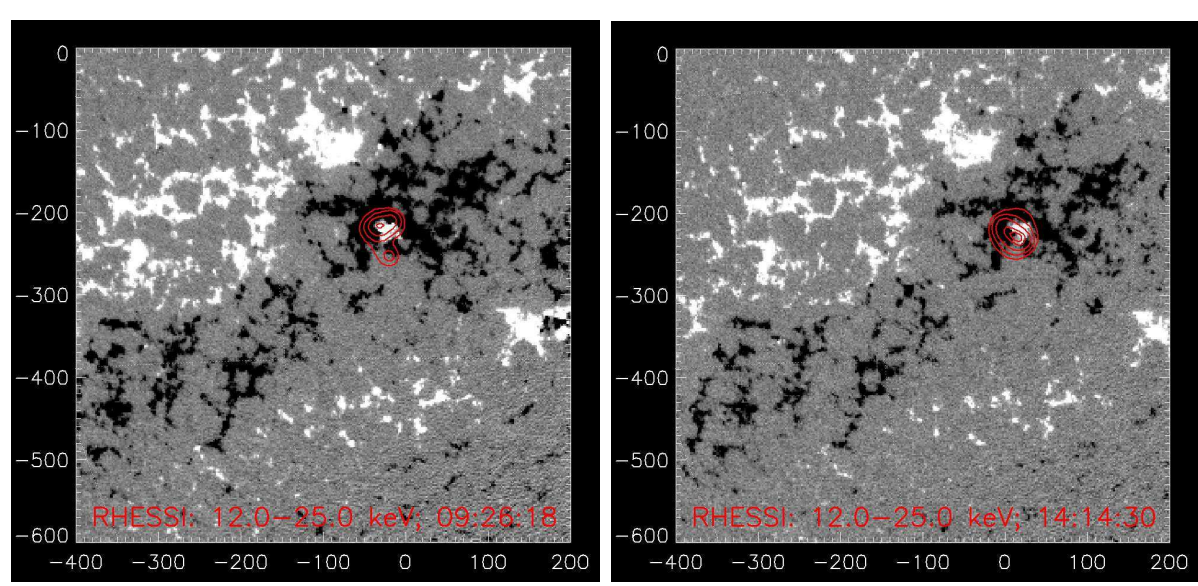

Figure 4. RHESSI HXR sources (contours, reconstructed by the Pixon method) in the $12-25 \mathrm{keV}$ spectral range superposed on the MDI magnetogram. The left panel shows the 09:26 UT event, the right panel the 14:14 UT event. There were no RHESSI data for the other two arcades. The coordinates are in units of arcsec.

occurred at 09:26 UT. The majority of the flux in Figure 5 came from the precursor structure. The hard channels peaked earlier than the soft channels (RHESSI: $25-50 \mathrm{keV}$ peaked at 09:25:04 UT, $12-25 \mathrm{keV}$ at 09:25:32 UT, $6-$ $12 \mathrm{keV}$ at 09:26:16 UT; the GOES channels peaked at 09:27:00 UT; and Mg XII peaked at 09:28:56 UT), which is consistent with the standard flare model.

We measured the light curves in the Mg XII $8.42 \AA$ line of the precursor structures and individual loops of the arcades (see Figure 6). The maximum intensity of the precursor structure exceeded the maximum intensity of the brightest loop by one order of magnitude and by two orders of the intensity of the faintest loop. The maximum intensity of the loops exponentially decreased with the distance from the precursor structure (see Figure 7). The intensity e-folding distance is $35 \pm 5 \mathrm{Mm}$.

To measure the plasma cooling time $(\tau)$, we approximated the light curves of the precursor structure and the loops by the following formula:

$$
I=I_{0} \mathrm{e}^{-t / \tau} .
$$

The cooling time was 4-6 min for the precursor structure and $15-35$ min for the loops (see Table 22). Although it is intuitive to interpret the longer lifetime of the loops as a sign of continuous heating, it is also possible to explain it as a pure cooling (see the Appendix).

\section{Discussion}

Some aspects of the observed arcades are unusual; the first one is the magnetic configuration in which the events occurred. The precursor occurred above the flux emergence region in a quadrupolar magnetic configuration, suitable for flaring reconnection (see Figure 8). It is clear that the flux emergence caused the 


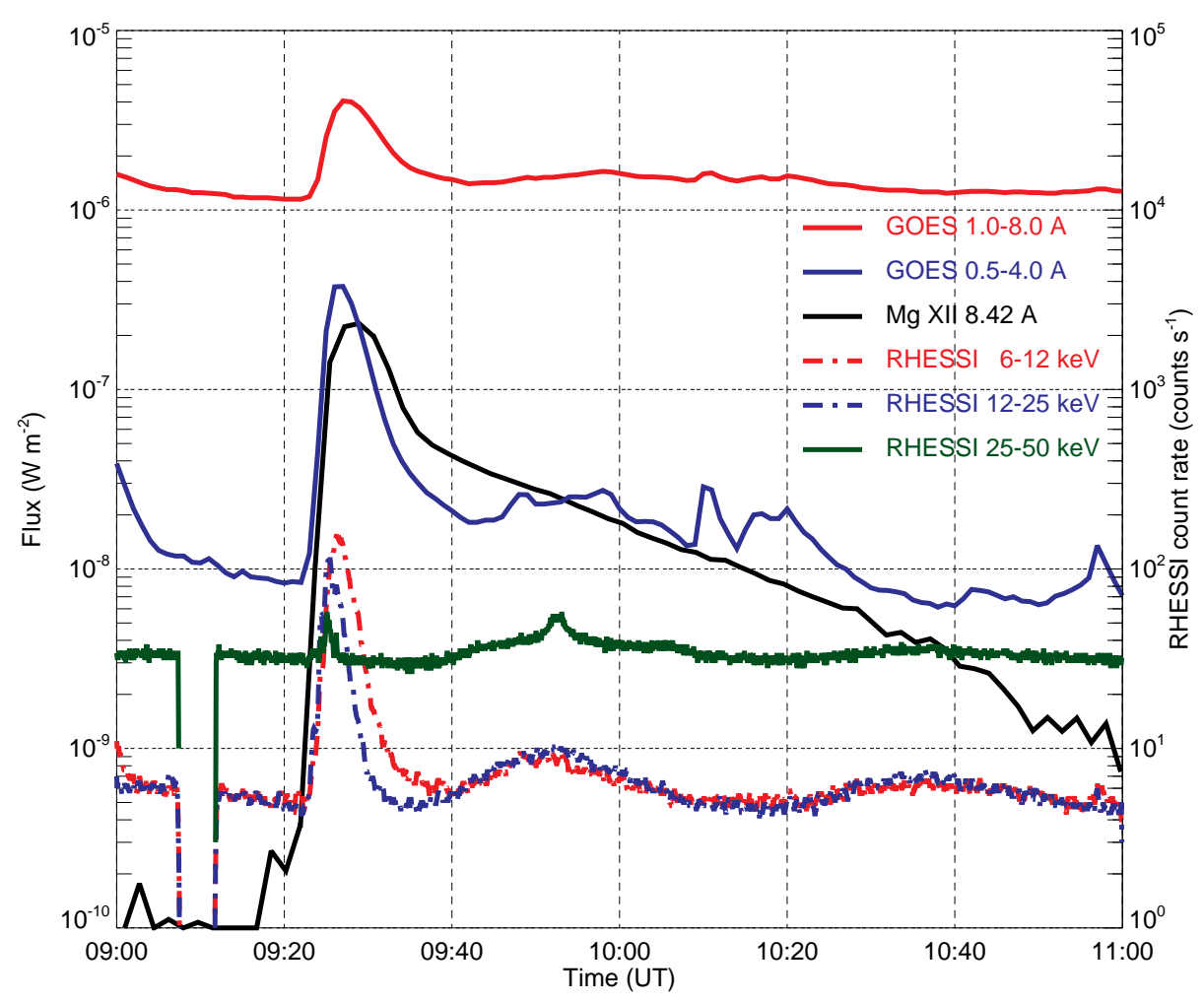

Figure 5. Light curves of the arcade that occurred on 28 February 2002 at 09:23 UT; we indicate the $1-8 \AA$ (red) and $0.5-4 \AA$ (blue) channels of GOES, Mg XII $8.42 \AA$ (black), $6-12 \mathrm{keV}$ (red dash-dotted), $12-25 \mathrm{keV}$ (blue dash-dotted), $25-50 \mathrm{keV}$ (green) channels of RHESSI.

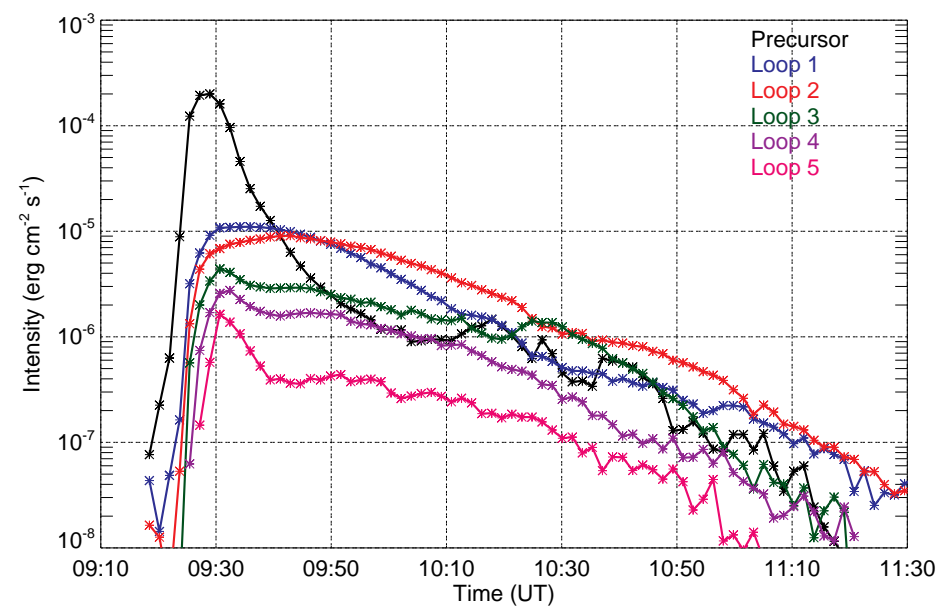

Figure 6. Light curves of the precursor structure and individual loops of the arcade in the Mg XII $8.42 \AA$ line. The arcade occurred after the flare on 28 February 2002 at 09:23 UT. The light curves of other arcades were similar to these curves. 


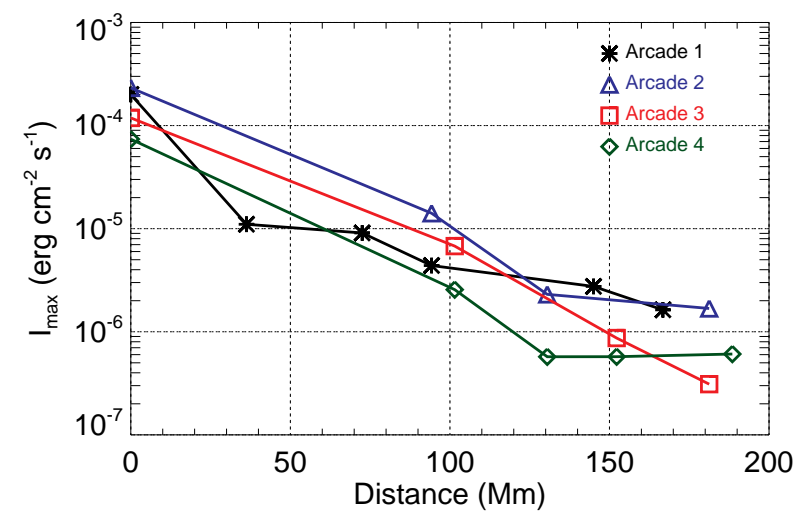

Figure 7. Maximum intensity of the loops observed in the Mg XII $8.42 \AA$ line as a function of the distance from the precursor structure.

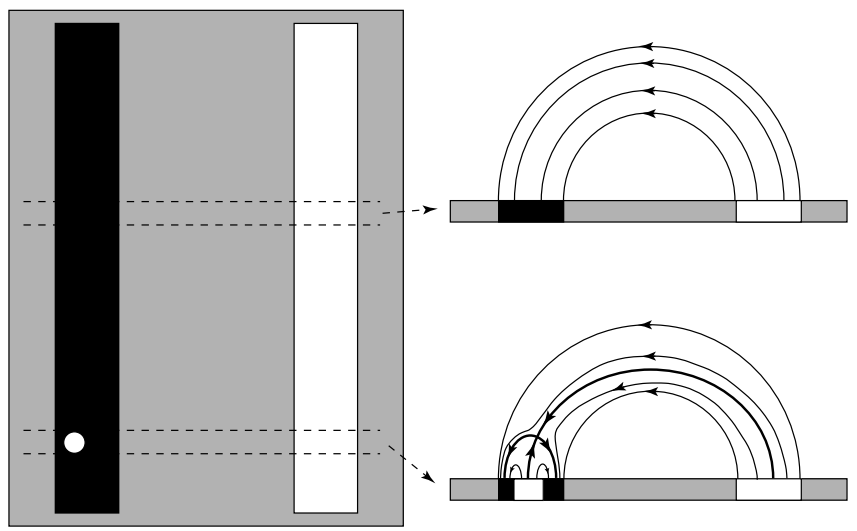

Figure 8. Schematic magnetic field model of the event. Black and white indicate negative and positive polarities, and gray means neutral polarity. The quasi-vertical current sheet above the arcade is not shown here (see Figure 9).

precursor reconnection. However, the arcade loops formed in a simple bipolar configuration (see Figure 8), without changes in their magnetic field structure.

Table 2. Decay times (measured in minutes) of the precursor structure and the loops of the arcade.

\begin{tabular}{ccccccc}
\hline Event & Precursor & Loop 1 & Loop 2 & Loop 3 & Loop 4 & Loop 5 \\
\hline 1 & 4.5 & 19 & 21 & 36 & 32 & 31 \\
2 & 6.6 & 21 & 24 & 24 & - & - \\
3 & 4.2 & 24 & 23 & 21 & - & - \\
4 & 5.4 & 16 & 19 & 24 & 10 & - \\
\hline
\end{tabular}


Second, the arcades formed in a wave-like manner, which indicates that waves might play a role in the phenomena. Third, there was a cold space (distance) between the hot loops. It is unclear why some loops of the arcade were heated and some were not. Neither the wave-like formation nor the cold space can be explained by the standard 2.5D flare model. We need an extension of the standard flare model into 3D, which will explain the observations.

To explain the observations, we propose that the current sheet existed above the loop apexes before the arcades ignited (see Figure 91). We think that the arcade evolution was the following:

i) The precursor structure was formed at the edge of the arcade (see Figure 97).

ii) The precursor launched an MHD wave, which propagated along the arcade (see Figure 9b).

iii) The MHD wave caused instabilities in the current sheet (see Figure 9r).

iv) The instabilities led to the heating of the underlying loops (see Figure $9 \mathrm{~d}$ ).
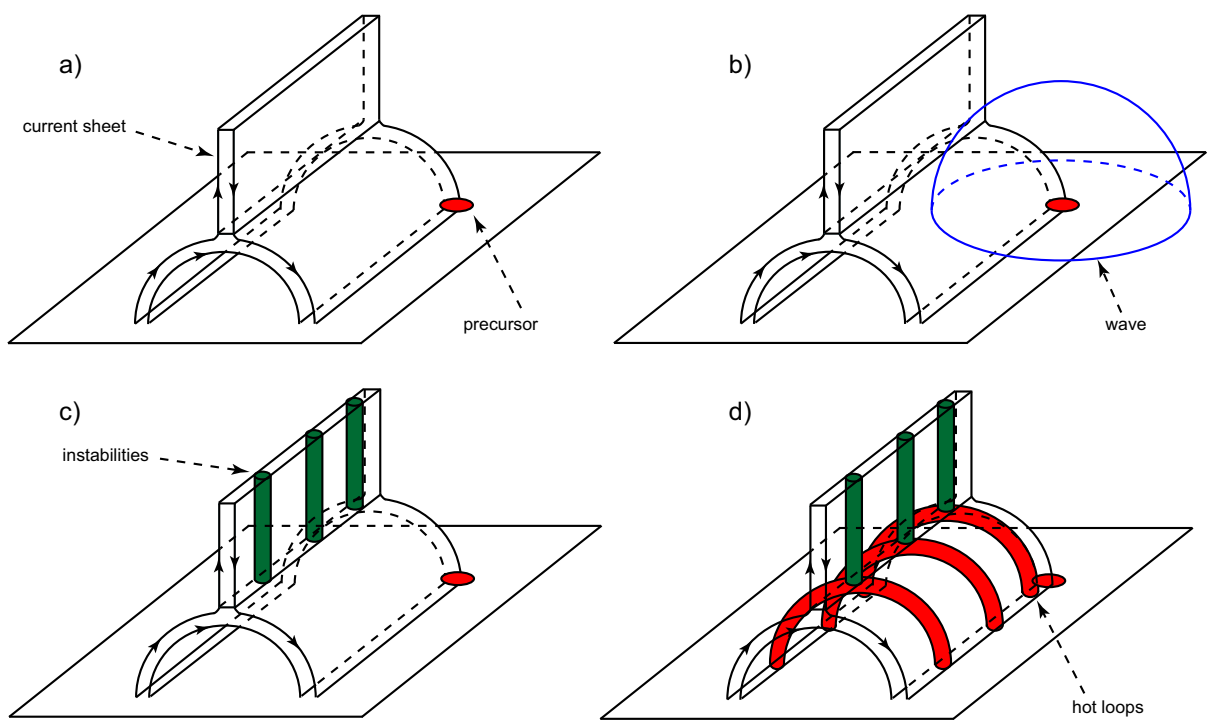

Figure 9. Schematic model of hot loop arcade formation.

Artemyev and Zimovets 2012) studied the unstable wave modes of a quasivertical $2.5 \mathrm{D}$ current sheet above an arcade with a sheared magnetic field. The studied waves propagated in the current sheet along the PIL. The development of the symmetrical sausage-mode waves modes to the local thinning and thickenings of the current sheet. Due to the conservation of magnetic flux, the current density is higher where the current sheet is thinner. This process intensifies the tearing instability Lapenta and Brackbill, 2000; Wiegelmann and Büchner, 2000; Lapenta, 2003) and modulates the efficiency of the energy release along the current sheet. Thus, the propagation of the unstable wave modes can lead to successive episodes of plasma heating in different loops of the arcade.

The characteristic distance between the two most effectively heated neighboring loops should approximately be equal to the wavelength $(\lambda)$ of the corre- 
sponding unstable wave mode. The characteristic speed of the triggering front propagating along the PIL should correspond to the group speed of the wave $\left(v_{\mathrm{g}}\right)$. The most appropriate unstable wave mode for the flaring loop arcades has the group speed (Artemyev and Zimovets, 2012)

$$
v_{\mathrm{g}} \approx \frac{v_{\mathrm{A}}}{4} \sqrt{\frac{\lambda}{L_{x}} \sin \varphi},
$$

where $v_{\mathrm{A}}$ is the Alfvén speed outside of the current sheet, $L_{x}$ is the vertical scale of the current sheet, and $\varphi$ is the shear angle.

Let us apply this theory to the observations. The impulsive energy release during the precursor process could initiate the unstable wave modes in the current sheet above the arcades. Artemyev and Zimovets (2012) predicted that the wavelength lies in the range from $0.1 \mathrm{Mm}$ to $1000 \mathrm{Mm}$. The observed value $\lambda \approx 50 \mathrm{Mm}$ corresponds to the collisionless mode 3 found in Artemyev and Zimovets (2012). From the observations, we estimate that $v_{\mathrm{g}} \approx 700 \mathrm{~km} \mathrm{~s}^{-1}$ and $\varphi \approx 30^{\circ}$ (see Figure 21). Thus, to satisfy Equation (2), $L_{x}$ should be

$$
L_{x} \approx 3 \times 10^{-3} v_{\mathrm{A}}^{2} \quad \mathrm{~km}
$$

Here, the Alfvén speed $v_{\mathrm{A}}$ is in $\mathrm{km} \mathrm{s}^{-1}$. For the estimation, we used the 'canonical' value for the solar corona $\left(v_{\mathrm{A}}=1000 \mathrm{~km} \mathrm{~s}^{-1}\right)$ and found that in the studied events $L_{x} \approx 3 \mathrm{Mm}$. This length is similar to what was found by Reid. Vilmer, and Kontar (2011) in their analysis of the coronal type III radio bursts. Finally, the estimated vertical length of the current sheet is $6-7$ orders of magnitude greater than the characteristic ion gyroradius in the solar corona (current sheet width), which is a favorable condition for magnetic reconnection and plasma heating.

Let us discuss other possible models. Emslie (1981) proposed that an increase in plasma pressure can increase the cross section of a hot flare loop. The hot loop will interact with a neighboring cold loop of the arcade and cause magnetic reconnection and heating in the cold loop. This process can propagate along the arcade like a domino effect. However, this mechanism cannot explain the cold space between the hot loops.

Nakariakov and Zimovets (2011) proposed that a slow magnetoacoustic wave that propagate along the arcade can trigger energy release in the current sheet above the arcade. The main difference with the model of Artemvev and Zimovets (2012) is the nature of the wave. In the model of Artemyev and Zimovets (2012) the wave propagates inside the current sheet, while in the model of Nakariakov and Zimovets (2011) the wave propagates along the arcade outside the current sheet. In our observations, a group of slow magnetoacoustic waves could be initiated by the precursor. The model of Nakariakov and Zimovets (2011) can explain the wave-like formation of a hot arcade, but it cannot easily explain the cold space between the hot loops.

Liu, Alexander, and Gilbert (2009) analyzed the flares in which a filament erupted asymmetrically along the PIL. In these events, the arcade should brighten in a wave-like manner. Although filaments can erupt several times in a row from 
the same active region (Archontis, Hood, and Tsinganos, 2014), the model fails to explain the cold space between the hot loops.

The model of Artemyev and Zimovets (2012) explains both the wave-like formation of the arcades and the cold space between the hot loops. The model gives a reasonable estimate of the current sheet vertical scale. The observed distance between the hot loops is consistent with the model. The Artemyev and Zimovets (2012) model fits our observations better than other models known to us. We hope that the reported observations will stimulate new theoretical investigations and observations of this type of solar flare phenomenon.

\section{Conclusions}

We presented the first observations of the formation of hot loop arcades in the hot monochromatic line. Thanks to the relatively high cadence of the Mg XII spectroheliograph, we saw the formation of the arcades in detail. At first, a small source of soft X-ray and hard X-ray emissions appeared at the edge of the future arcade. Then the arcade brightened in a wave-like manner. The wave speed was about $700 \mathrm{~km} \mathrm{~s}^{-1}$, which is the same order of magnitude as the MHD coronal wave speed. The arcades occurred four times in a row in the same place under the same conditions, following the same scenario.

We interpreted the observations in terms of the MHD waves. We think that the current sheet existed above the loop apexes before the arcade ignited. The precursor launched an MHD wave, which triggered instabilities in the current sheet. The instabilities led to the reconnection and loop heating.

Our interpretation is consistent with previous theoretical works (Somov and Syrovatskii, 1982; Artemyev and Zimovets, 2012). The observations show that flares are essentially a 3D processes 2D models do not explain all their aspects. The observations also point out that MHD waves could play an important role in flare processes; they could transfer energy and trigger reconnection.

Acknowledgments We are grateful to Boris Somov and Anton Artemyev for their invaluable help. This work was supported by a grant from the Russian Foundation of Basic Research (grant 14-02-00945) and by the Program No. 22 for fundamental research of the Presidium of the Russian Academy of Sciences.

\section{Appendix}

\section{Cooling times}

The loops of the arcade kept their high temperature for a long time (see Figure 6). This could be interpreted as a sign of the continuous loop heating. To verify this conclusion, we here estimate the plasma cooling time in the absence of heating, and compare it with the measured values.

There are two mechanisms of loop cooling: conductive and radiative. At high temperatures $(\approx 10 \mathrm{MK})$, the conductive cooling dominates radiative cooling. 




Figure 10. Temperature response functions of the Mg XII spectroheliograph (solid line) and EIT 195 Å telescope (dashed line).

To estimate the conductive cooling time $\left(\tau_{\text {rand }}\right)$. we use the formula (Culhane et al., 1994)

$$
\tau_{\text {cond }}=\frac{21 n_{\mathrm{e}} k_{\mathrm{B}} L^{2}}{5 \kappa T^{5 / 2}},
$$

where $\kappa=9.2 \times 10^{-7} \mathrm{erg} \mathrm{s}^{-1} \mathrm{~cm}^{-1} \mathrm{~K}^{-7 / 2}$ is the Spitzer conductivity, $n_{\mathrm{e}}$ is the electron density, $k_{\mathrm{B}}$ is the Boltzmann constant, $L$ is the loop length, and $T$ is the loop temperature.

To estimate the arcade temperature, we compared Mg XII and EIT $195 \AA$ images. The EIT $195 \AA$ channel is sensitive to $1 \mathrm{MK}$ and $16 \mathrm{MK}$ plasma (see Figure (10). The Mg XII spectroheliograph is sensitive to plasmas hotter than 5 MK (see Figure 10). Since we see the precursor structure in both EIT $195 \AA$ and Mg XII channels, the precursor temperature should be about 15 MK. Since we were able to see the arcade in the Mg XII line but not in the EIT images, the loop temperature should lie in the range of $5-10 \mathrm{MK}$.

To estimate the precursor electron density, we estimated its temperature $(T)$ and emission measure (EM) under isothermal approximation using the filter ratio method with EIT $195 \AA$ and Mg XII fluxes. The result is $T \approx 14 \mathrm{MK}$ and $\mathrm{EM} \approx 8.8 \cdot 10^{46} \mathrm{~cm}^{-3}$. The precursor structure had a length of $L \approx 20 \mathrm{Mm}$ and the width of the EIT pixel. However, since loops in the AIA images have the width of the AIA pixel $(r=0.44 \mathrm{Mm})$, we think that the AIA pixel size is a more reasonable estimate for the loop width than the EIT pixel size. Now, we can estimate the precursor electron density:

$$
\mathrm{EM}=n_{\mathrm{e}}^{2} \pi r^{2} L \Rightarrow n_{\mathrm{e}}=\sqrt{\frac{\mathrm{EM}}{\pi r^{2} L}} \approx 8.6 \cdot 10^{10} \mathrm{~cm}^{-3} .
$$

Unfortunately, we have only Mg XII observations for the flaring loops, so that there is no way to measure the emission measure and temperature of their plasma separately using the filter ratio. However, we can estimate their electron density from 


$$
I=G(T) n_{\mathrm{e}}^{2} \pi r^{2} L \leq G_{\max } n_{\mathrm{e}}^{2} \pi r^{2} L,
$$

namely,

$$
n_{\mathrm{e}} \geq \sqrt{\frac{I}{G_{\max } \pi r^{2} L}} \approx 1.8 \cdot 10^{10} \mathrm{~cm}^{-3}
$$

where $I$ is the emission intensity of the loops on the Mg XII images, $G(T)$ is the temperature response function of the $\mathrm{Mg}$ XII spectroheliograph, and $G_{\max }$ is the maximum value of $G(T)$. For the sake of estimation, we used $n_{\mathrm{e}}=10^{10} \mathrm{~cm}^{-3}$ and $T=10 \mathrm{MK}$ for the loops of the arcade.

For the loops, we obtained $\tau_{\text {cond }}=100 \min \left(n_{\mathrm{e}}=10^{10} \mathrm{~cm}^{-3}, T=10 \mathrm{MK}\right.$, and $L=170 \mathrm{Mm}$ ). This is of the same order of magnitude as the measured values, $20-30 \mathrm{~min}$. Furthermore, slight changes of the $n_{\mathrm{e}}$ and $T$ values can make the agreement better. This means that it is possible that the loop heating was impulsive and long lifetime of the loops was due to their large size.

For the precursor, we obtained $\tau_{\text {cond }}=5 \min \left(n_{\mathrm{e}}=8.6 \cdot 10^{10} \mathrm{~cm}^{-3}, T=\right.$ $14 \mathrm{MK}$, and $L=20 \mathrm{Mm}$ ), which coincides with the observed values. This means that it is possible that the heating of the precursor structure was impulsive.

It may be surprising that the long decay of the Mg XII light curves can be interpreted as pure cooling. However, our estimate is very rough; it is valid in the temperature range $6.8<\log T<7.3$, where the response function of $\mathrm{Mg}$ XII channel does not change much. Therefore, pure cooling is only an option, but not a fact.

Furthermore, other mechanisms could decrease the loop intensity; for example by mass draining. The mass draining could play a significant role in the late phase of flare decay (Bradshaw and Cargill, 2010). The draining will decrease the electron density, and therefore the loop intensity will decrease faster. Also, according to Equation (4), the loops will cool faster. If the draining was present, then the hot loops would require continuous heating to support their long decay times.

\section{References}

Archontis, V., Hood, A.W., Tsinganos, K.: 2014, Recurrent explosive eruptions and the "sigmoid-to-arcade" transformation in the Sun driven by dynamical magnetic flux emergence. Astrophys. J. Lett. 786, L21. DOI ADS

Artemyev, A., Zimovets, I.: 2012, Stability of current sheets in the solar corona. Solar Phys. 277, 283. DOI ADS

Benz, A.O.: 2008, Flare observations. Living Rev. in Solar Phys. 5(1). DOI http://www. livingreviews.org/Irsp-2008-1.

Bogachev, S.A., Somov, B.V., Kosugi, T., Sakao, T.: 2005, The motions of the hard X-ray sources in solar flares: Images and statistics. Astrophys. J. 630, 561. DOI. ADS

Bradshaw, S.J., Cargill, P.J.: 2010, The cooling of coronal plasmas. III. Enthalpy transfer as a mechanism for energy loss. Astrophys. J. 717, 163. DOI ADS

Carmichael, H.: 1964, A process for flares. In: Ness, W.N. (ed.) AAS-NASA Symposium on the Physics of Solar Flares, NASA SP-50, 451. ADS

Culhane, J.L., Phillips, A.T., Inda-Koide, M., Kosugi, T., Fludra, A., Kurokawa, H., Makishima, K., Pike, C.D., Sakao, T., Sakurai, T.: 1994, YOHKOH observations of the creation of high-temperature plasma in the flare of 16 December 1991. Solar Phys. 153, 307. DOI ADS 
Delaboudinière, J., Artzner, G.E., Brunaud, J., Gabriel, A.H., Hochedez, J.F., et al.: 1995, EIT: Extreme-Ultraviolet Imaging Telescope for the SOHO mission. Solar Phys. 162, 291. DOI ADS

Domingo, V., Fleck, B., Poland, A.I.: 1995, The SOHO mission: An overview. Solar Phys. 162, 1. DOI ADS

Emslie, A.G.: 1981, An interacting loop model for solar flare bursts. Astrophys. J. Lett. 22, 41. ADS

Fletcher, L., Dennis, B.R., Hudson, H.S., Krucker, S., Phillips, K., Veronig, A., et al.: 2011, An observational overview of solar flares. Space Sci. Rev. 159, 19. DOI. ADS

Golub, L., Deluca, E., Austin, G., Bookbinder, J., Caldwell, D., Cheimets, P., et al.: 2007, The X-Ray Telescope (XRT) for the Hinode mission. Solar Phys. 243, 63. DOI ADS.

Grigis, P.C., Benz, A.O.: 2005, The spectral evolution of impulsive solar X-ray flares. II. Comparison of observations with models. Astron. Astrophys. 434, 1173. DOI ADS

Hirayama, T.: 1974, Theoretical model of flares and prominences. I: Evaporating flare model. Solar Phys. 34, 323. DOI. ADS

Jing, J., Lee, J., Liu, C., Gary, D.E., Wang, H.: 2007, Hard X-ray intensity distribution along $\mathrm{H} \alpha$ ribbons. Astrophys. J. Lett. 664, L127. DOI ADS

Kopp, R.A., Pneuman, G.W.: 1976, Magnetic reconnection in the corona and the loop prominence phenomenon. Solar Phys. 50, 85. DOI ADS

Krucker, S., Hudson, H.S., Jeffrey, N.L.S., Battaglia, M., Kontar, E.P., Benz, A.O., Csillaghy, A., Lin, R.P.: 2011, High-resolution imaging of solar flare ribbons and its implication on the thick-target beam model. Astrophys. J. 739, 96. DOI. ADS.

Lapenta, G.: 2003, A new paradigm for 3D collisionless magnetic reconnection. Space Sci. Rev. 107, 167. DOI ADS

Lapenta, G., Brackbill, J.U.: 2000, 3D reconnection due to oblique modes: a simulation of harris current sheets. Nonlinear Proc. in Geophys. 7, 151. DOI http://www. nonlin-processes-geophys.net/7/151/2000/.

Lemen, J.R., Title, A.M., Akin, D.J., Boerner, P.F., Chou, C., Drake, J.F., et al.: 2011, The Atmospheric Imaging Assembly (AIA) on the Solar Dynamics Observatory (SDO). Solar Phys., 172. DOI ADS

Lin, R.P., Dennis, B.R., Hurford, G.J., Smith, D.M., Zehnder, A., Harvey, P.R., et al.: 2002, The Reuven Ramaty High-Energy Solar Spectroscopic Imager (RHESSI). Solar Phys. 210, 3. DOI ADS

Liu, C., Lee, J., Yurchyshyn, V., Deng, N., Cho, K.S., Karlický, M., Wang, H.: 2007, The eruption from a sigmoidal solar active region on 2005 May 13. Astrophys. J. 669, 1372. DOI ADS

Liu, R., Alexander, D., Gilbert, H.R.: 2009, Asymmetric eruptive filaments. Astrophys. J. 691, 1079. DOI ADS

Masuda, S., Kosugi, T., Hudson, H.S.: 2001, A hard X-ray two-ribbon flare observed with Yohkoh/HXT. Solar Phys. 204, 55. DOI ADS

McLaughlin, J.A., Hood, A.W., de Moortel, I.: 2011, Review article: MHD wave propagation near coronal null points of magnetic fields. Space Sci. Rev. 158, 205. DOI ADS

Nakariakov, V.M., Zimovets, I.V.: 2011, Slow magnetoacoustic waves in two-ribbon flares. Astrophys. J. Lett. 730, L27. DOI ADS

Nakariakov, V.M., Foullon, C., Verwichte, E., Young, N.P.: 2006, Quasi-periodic modulation of solar and stellar flaring emission by magnetohydrodynamic oscillations in a nearby loop. Astron. Astrophys. 452, 343. DOI ADS

Oraevsky, V.N., Sobelman, I.I.: 2002, Comprehensive studies of solar activity on the CORONAS-F satellite. Astron. Lett. 28, 401. DOI ADS

Parenti, S., Reale, F., Reeves, K.K.: 2010, Post-flare evolution of AR 10923 with Hinode/XRT. Astron. Astrophys. 517, A41. DOI ADS

Reale, F., Guarrasi, M., Testa, P., DeLuca, E.E., Peres, G., Golub, L.: 2011, Solar Dynamics Observatory discovers thin high temperature strands in coronal active regions. Astrophys. J. Lett. 736, L16. DOI ADS

Reid, H.A.S., Vilmer, N., Kontar, E.P.: 2011, Characteristics of the flare acceleration region derived from simultaneous hard X-ray and radio observations. Astron. Astrophys. 529, A66. DOI ADS

Reva, A., Shestov, S., Bogachev, S., Kuzin, S.: 2012, Investigation of hot X-ray points (HXPs) using spectroheliograph Mg XII experiment data from CORONAS-F/SPIRIT. Solar Phys. 276, 97. DOI ADS 
Scherrer, P.H., Bogart, R.S., Bush, R.I., Hoeksema, J.T., Kosovichev, A.G., Schou, J., et al.: 1995, The Solar Oscillations Investigation - Michelson Doppler Imager. Solar Phys. 162, 129. DOI ADS

Somov, B.V., Syrovatskii, S.I.: 1982, Thermal trigger for solar flares and coronal loops formation. Solar Phys. 75, 237. DOI ADS

Sturrock, P.A.: 1966, Model of the high-energy phase of solar flares. Nature 211, 695. DOI ADS

Testa, P., Reale, F.: 2012, Hinode/EIS spectroscopic validation of very hot plasma imaged with the Solar Dynamics Observatory in non-flaring active region cores. Astrophys. J. Lett. 750, L10. DOI ADS

Tripathi, D., Isobe, H., Mason, H.E.: 2006, On the propagation of brightening after filament/prominence eruptions, as seen by SoHO-EIT. Astron. Astrophys. 453, 1111. DOI ADS

Tsuneta, S., Acton, L., Bruner, M., Lemen, J., Brown, W., Caravalho, R., Catura, R., Freeland, S., Jurcevich, B., Owens, J.: 1991, The soft X-ray telescope for the SOLAR-A mission. Solar Phys. 136, 37. DOI ADS.

Urnov, A.M., Shestov, S.V., Bogachev, S.A., Goryaev, F.F., Zhitnik, I.A., Kuzin, S.V.: 2007, On the spatial and temporal characteristics and formation mechanisms of soft X-ray emission in the solar corona. Astron. Lett. 33, 396. DOI ADS.

Vorpahl, J.A.: 1976, The triggering and subsequent development of a solar flare. Astrophys. J. 205, 868. DOI ADS

Warren, H.P., Winebarger, A.R., Brooks, D.H.: 2012, A systematic survey of high-temperature emission in solar active regions. Astrophys. J. 759, 141. DOI ADS

Warren, H.P., Bookbinder, J.A., Forbes, T.G., Golub, L., Hudson, H.S., Reeves, K., Warshall, A.: 1999, TRACE and Yohkoh observations of high-temperature plasma in a two-ribbon limb flare. Astrophys. J. Lett. 527, L121. DOI ADS

Wiegelmann, T., Büchner, J.: 2000, Kinetic simulations of the coupling between current instabilities and reconnection in thin current sheets. Nonlinear Proc. in Geophys. 7, 141. DOI http://www.nonlin-processes-geophys.net/7/141/2000/.

Zhitnik, I.A., Bougaenko, O.I., Delaboudiniere, J.-P., Ignatiev, A.P., Korneev, V.V., Krutov, V.V., et al.: 2002, SPIRIT X-ray telescope/spectroheliometer results. In: Wilson, A. (ed.) Solar Variability: From Core to Outer Frontiers, ESA SP-506, 915. ADS

Zhitnik, I.A., Bugaenko, O.I., Ignat'ev, A.P., Krutov, V.V., Kuzin, S.V., Mitrofanov, A.V., et al.: 2003, Dynamic $10 \mathrm{MK}$ plasma structures observed in monochromatic full-Sun images by the SPIRIT spectroheliograph on the CORONAS-F mission. Mon. Not. Roy. Astron. Soc. 338, 67. DOI ADS 